\title{
Factores asociados al trato humanizado del personal de enfermería a usuarios ingresados en el hospital de especialidades José Carrasco Arteaga. Cuenca - 2019
}

\section{Factors associated with the humanized treatment of nursing staff to users admitted to the José Carrasco Arteaga specialty hospital. Cuenca - 2019}

\author{
Lucia Gabriela Chin Orbes*1, Johanna Melina Roldan Morales ${ }^{1}$, Marcia Yolanda Cobos Alvarracín ${ }^{1}$, Johnny \\ Fabián Vizuela Carpio ${ }^{1}$, Paola Valeria Rivera Asitimbay ${ }^{1}$ \\ ${ }^{1}$ Universidad Católica de Cuenca, Ecuador \\ *lgchino59@est.ucacue.edu.ec
}

DOI: https://doi.org/10.26871/killkanasalud.v4i4.756

\begin{abstract}
Resumen
Introducción: El cuidado humanizado es una forma de expresión, con otro ser y con el mundo, puede ser demostrado de manera afectiva y practicado de forma interpersonal, consta de factores que puede satisfacer las necesidades de los usuarios hospitalizados. Objetivo: Determinar los factores asociados al trato humanizado del personal de enfermería en usuarios ingresados en el Hospital de especialidades José Carrasco Arteaga. Metodología: Estudio cuantitativo, correlacional, de corte transversal, el tamaño de la muestra fue de 384 pacientes. Se utilizaron dos instrumentos, que evaluaron la percepción del cuidado humanizado de enfermería, clasificación de pacientes según su complejidad y recolección de variables a relacionar. Resultados: Al obtener los resultados, en cuanto a la edad prevaleció el adulto con $62,8 \%$, prevalece el sexo masculino con 55,7\%, en cuanto al trato humanizado la mayor parte de los encuestados dieron una respuesta de siempre reciben un trato humanizado con un porcentaje de $97,4 \%$, mientras que el $2,6 \%$ dieron una respuesta de casi siempre perciben un trato humanizado, al obtener las medidas de asociación entre trato humanizado y edad, sexo, estado civil, nivel de instrucción, etnia, residencia, turno de servicio, días de hospitalización, área de servicio y complejidad del paciente no existe una asociación significativa. Conclusión: En el presente estudio se obtuvo una asociación significativa en la variable tipo de seguro con relación al trato humanizado, mientras que en las demás variables no se obtuvo una asociación significativa.
\end{abstract}

Palabras clave: humanización de la atención, atención al paciente, atención de enfermería.

\begin{abstract}
Introduction: Humanized care is a form of expression, with another being and with the world, it can be demonstrated affectively and practiced interpersonally, it consists of factors that can meet the needs of hospitalized users. Objective: To determine the factors associated with the humane treatment of the nursing staff in users admitted to the José Carrasco Arteaga Specialty Hospital. Methodology: It is a quantitative, correlational, cross-sectional study, the sample size was 384 patients. Two instruments were used, the first one that assessed the perception of humanized nursing care, the second instrument helped us to classify patients according to their complexity. Results: When obtaining the results, as for the age, the adult prevailed with $62.8 \%$, the male sex prevails with $55.7 \%$, as for the humanized treatment most of the respondents gave a response of always receiving a humanized treatment with a percentage of $97.4 \%$, while $2.6 \%$ gave a response almost always perceived humanized treatment, obtaining the measures of association between humanized treatment and age, sex, marital status, level of education, ethnicity, residence, shift of service, days of hospitalization, service área and complexity of the patient there is no significant association. Conclusion: In the present study a significant association was obtained in the type of insurance variable in relation to humanized treatment, while in the other variables a significant association was not obtained.
\end{abstract}

Keywords: humanization of care, patient care, nursing care . 


\section{Introducción}

El hombre como ser social está sujeto a relaciones interpersonales que se promueven entre personas que se conocen o no, estas juegan un rol importante en la actividad humana y están mediadas por la comunicación. En enfermería, se establecen relaciones con el paciente y familiares, de modo que le permita a la enfermera favorecer la relación terapéutica, satisfacer las necesidades del paciente y brindar cuidados de enfermería. El cuidado como centro de atención de la enfermería, se describe también como la relación que se da entre enfermera-paciente con el fin de promover su salud, prevenir, diagnosticar, tratar y rehabilitar las enfermedades que puedan padecer.

El profesional de enfermería debe estar en capacidad de brindar cuidados a todas las personas, independientemente de su condición; esta atención debe garantizar el bienestar y la seguridad de las personas, preservando su salud. Para lograr su objetivo debe estar en permanente desarrollo y fortalecer actitudes y valores que permitan la humanización en su quehacer cotidiano, siendo reflexivo, crítico, comprometido, humanista, solidario, respetuoso, honesto, creativo, participativo y responsable para atender al individuo, en un sistema de salud que busca satisfacer las necesidades de la población a cuidar ${ }^{2}$.

La enfermería se centra en el cuidado de la salud y de la vida, hacia esa pretensión se encaminan los esfuerzos y trabajos que se realizan para la atención de los sujetos de cuidado; la persona, la familia y la comunidad. Los escenarios de cuidado se dan en la vida cotidiana, en tanto que es ahí en donde se desarrollan las actividades diarias de las personas. Por otro lado, los cuidados también se dan en el ambiente hospitalario, pero no es ese el único espacio en donde se desarrolla la esencia de la enfermería. ${ }^{3}$

Considerando que la enfermera es la encargada de brindar el cuidado directo al paciente hospitalizado, este cuidado debe ser basado en el bienestar humano y velando por la satisfacción de los usuarios; en la actualidad se cree que se ha deshumanizado día a día, no es extraño que a los pacientes se les reconoce por el número de cama, o por su patología, en los hospitales ha sufrido un deterioro debido a las múltiples funciones que debe desempeñar la enfermera. ${ }^{4}$

En México, en 2016, el estándar de calidad para el indicador de tratamiento digno por parte del personal de enfermería disminuyó en comparación con 2012, del $97 \%$ al $96.8 \%$. Para las instituciones de salud, no ha sido una tarea fácil cumplir con los estándares de calidad para mejorar la atención a la población, y el cumplimiento de este indicador no ha sido la excepción, ya que se ve afectado por diferentes factores, tales como: falta de recursos humanos en atención directa a pacientes; apoyo financiero limitado para el suministro de material de trabajo, entre otros. ${ }^{5}$

En Lima - Perú en el año 2015, Guerrero - Ramírez $\mathrm{R}$, et al., mostró que la percepción de trato humanizado es regular en un $52 \%$ de la población, bajo en el $22 \%$ y apenas un $26 \%$ tiene una alta percepción. Al evaluar las dimensiones de trato humanizado prevalece una percepción regular con valores que oscilan entre el $45 \%$ y el $95 \%{ }^{6}$

En Chile, Ramos GS, et al., reporta que del total de población enfermero/a el 48,33\% menciona tener una menor percepción de entrega de trato humanizado, lo que podría indicar que los pacientes no reciben el trato humanizado óptimo, debido a que el personal de enfermería es responsable del cuidado de varios pacientes y mayor percepción el $51,67 \% .^{4}$

En Chile, Landman C. et al., en su estudio sobre satisfacción usuaria respecto a competencia de comunicación del profesional de enfermería, presenta los resultados respecto al grado de satisfacción global de la comunicación del profesional de enfermería percibida por los pacientes muestran índices positivos en las tres instituciones estudiadas, los valores de satisfacción oscilan entre 73 y $100 \%$, considerando los valores de satisfecho y muy satisfecho. Al analizar los datos de acuerdo a las variables de caracterización, las mujeres muestran mayores niveles de insatisfacción en relación a los hombres, siendo solo ellas quienes manifestaron un mayor grado de insatisfacción. ${ }^{7}$

El Modelo de Atención Integral en Salud (MAIS) en Ecuador busca que las actividades desarrolladas en los establecimientos de la Red Publica Integral de Salud y la Red Complementaria tengan resultados de impacto social. ${ }^{8}$ En la actualidad es importante que los profesionales de enfermería incorporen medidas que satisfagan a los usuarios en un alto nivel de trato humanizado y de esta manera aportar el proceso de recuperación del paciente y brindar la seguridad a su familia, demostrando la ética y profesionalismo al brindar una atención. Para los pacientes es importante que la comunicación y la visualización por parte del personal de enfermería debe ser única y autónoma, por otra parte, el paciente tiene derecho a ser informado y a su vez tomar cualquier decisión sobre su salud, estas medidas serán respetadas de tal modo que exista una relación de persona a persona. El objetivo de la presente investigación es determinar los factores asociados al trato humanizado del personal de enfermería en usuarios ingresados en el Hospital de especialidades José Carrasco.

\section{Materiales y metodos}

La presente investigación tiene un enfoque cuantitativo, correlacional y de corte transversal, la población de estudio estuvo conformada por pacientes hospitalizados en el Hospital de especialidad José Carrasco Arteaga en el periodo agosto - septiembre 2019. Para el cálculo el tamaño de la muestra se consideró sobre la base del $95 \%$ del nivel de confianza y $5 \%$ de error de inferencia obteniendo muestra de 384 usuarios. Al no poseer un listado de pacientes previamente establecido se aplicó un muestreo no probabilístico por conveniencia, en donde se captaron pacientes que cumplan criterios de inclusión según el orden de ingreso al hospital hasta completar el número de participantes que se requiere en el estudio, se analizó mediante el programa SPSS. 
Se incluyeron en el presente estudio uuarios mayores de 18 años con funciones cognitivas normales con una estadía mínima de $24 \mathrm{~h}$ y que acepten ser parte de la investigación y firmen el consentimiento informado.

Para la recolección de información se utilizó el instrumento que mide la Percepción del Cuidado Humanizado de Enfermería (PCHE), el mismo que estuvo conformado por 32 preguntas en escala de Likert con valoraciones de 1 a 4 , agrupadas a tres dimensiones que son: 1) Priorizar el sujeto de cuidado (la persona), 2) Apertura a la comunicación para proporcionar educación en salud a la persona. (apoyo emocional), 3) Cualidades de hacer de enfermería (apoyo físico).

Permite valorar los siguientes niveles:

- Siempre ser percibe una relación de cuidado de enfermería (puntuación de la escala entre 104.1- 128)

- Casi siempre se percibe una relación de cuidado humanizado de enfermería (puntuación de la escala entre 80.1- 104)

- Algunas veces se percibe una relación de cuidado humanizado de enfermería (puntuación de la escala 56.180)

- Nunca se percibe una relación de cuidado humanizado de enfermería (puntuación de la escala entre 32-56). ${ }^{9}$

Para medir la complejidad del paciente se aplicó el instrumento de PERROCA por parte de los profesionales de enfermería que realizaban asistencia directa al paciente en los diferentes turnos y áreas de servicio, para clasificación de pacientes el instrumento estuvo constituido por nueve áreas de cuidado se describe a continuación:

Planeamiento y coordinación del proceso de cuidar, investigación y monitoreo, cuidado corporal y eliminación, cuidados de la piel y mucosas, nutrición e hidratación, locomoción y actividad, terapéutica, soporte emocional y educación en salud. ${ }^{10}$

Posterior a la recolección de la información los datos fueron ingresados en Excel 2016 para el desarrollo de la base de datos y finalmente los análisis fueron efectuados en el software estadístico SPSS versión 15, las variables cuantitativas se analizaron en base a medidas de frecuencia y porcentajes. Para encontrar una relación entra la variable complejidad del paciente se aplicó Odss Ratio con sus respectivos intervalos de confianza y Chi cuadrado como estadístico de asociación con un valor de $\mathrm{p}=>0,05$.

\section{Resultados}

Se recolectaron datos de 384 pacientes que se encontraban hospitalizados en el Hospital de Especialidades y Médicas José Carrasco Arteaga, los datos se analizaron en base a estadística descriptiva utilizando medidas de frecuencia y porcentaje, para el análisis correlacional se aplicó Odds Ratio como medida de estimación de riesgo con sus respectivos intervalos de confianza y Chi cuadrado como medida de significancia estadística, considerando un valor $\mathrm{p}<0,05$ para comprobación de hipótesis de trabajo.
En la tabla 1, indica que la edad más prevalente en pacientes hospitalizados estuvo comprendida entre adulto joven y adulto siendo el $95.6 \%$ y adulto mayor con el 4,4\%. Así también en relación al sexo los pacientes masculinos estuvieron más días hospitalizados que las mujeres, en relación al estado civil el $57,3 \%$ de los pacientes fueron casados el $42,7 \%$ de este grupo estuvieron distribuidos entre soltero, divorciados y viudos. En relación al nivel de instrucción se pudo observar que la secundaria fue de $62,2 \%$ siendo el mayor porcentaje mientras que el $0,8 \%$ estuvo relacionado con formación de cuarto nivel, en relación a la etnia se manifiesta que $98,9 \%$ fueron mestizos y se manifiesta que el $0,6 \%$ fue indígena y afro ecuatoriano y el $0,5 \%$ fue montubio.

Tabla 1: Distribución de la población según variables socio demográficas $(\mathrm{n}=384)$

\begin{tabular}{|c|c|c|}
\hline Variable & $\begin{array}{c}\text { Frecuencia } \\
\text { N }\end{array}$ & Porcentaje \% \\
\hline \multicolumn{3}{|l|}{ Edad } \\
\hline Adulto Joven & 126 & $32,8 \%$ \\
\hline Adulto & 241 & $62,8 \%$ \\
\hline Adulto Mayor & 17 & $4,4 \%$ \\
\hline \multicolumn{3}{|l|}{ Sexo } \\
\hline Hombre & 214 & $55,7 \%$ \\
\hline Mujer & 170 & $44,3 \%$ \\
\hline \multicolumn{3}{|l|}{ Estado Civil } \\
\hline Soltero & 47 & $12,2 \%$ \\
\hline Casado & 220 & $57,3 \%$ \\
\hline Unión Libre & 64 & $16,7 \%$ \\
\hline Divorciado & 38 & $9,9 \%$ \\
\hline Viudo & 15 & $3,9 \%$ \\
\hline \multicolumn{3}{|c|}{ Nivel de Instrucción } \\
\hline Analfabeto & 14 & $3,6 \%$ \\
\hline Primaria & 93 & $24,2 \%$ \\
\hline Secundaria & 239 & $62,2 \%$ \\
\hline Tercer Nivel & 35 & $9,2 \%$ \\
\hline Cuarto Nivel & 3 & $0,8 \%$ \\
\hline \multicolumn{3}{|l|}{ Etnia } \\
\hline Indígena & 1 & $0,3 \%$ \\
\hline Afro ecuatoriano & 2 & $0,5 \%$ \\
\hline Montubio & 1 & $0,3 \%$ \\
\hline Mestizo & 380 & $98,9 \%$ \\
\hline \multicolumn{3}{|l|}{ Residencia } \\
\hline Sierra & 242 & $63,0 \%$ \\
\hline Costa & 108 & $28,1 \%$ \\
\hline Oriente & 34 & $8,9 \%$ \\
\hline
\end{tabular}

Fuente: Base de datos SPSS

Elaborado por: Autores

En la tabla 2 se evidencia que el tipo de seguro que más prevalece es el seguro general con el $84,9 \%$, y otros seguros con el $15,1 \%$, en relación al número de pacientes atendidos en diferentes turno se puedo observar que $69,3 \%$ fueron atendidos en la tarde y noche y el $30,7 \%$, fueron atendido en el turno matutino, en cuanto a los días de hospitalización el $42 \%$ estuvieron entre dos y tres días, mientras que el $58 \%$ estuvieron hospitalizados entre cuatro y más días. 
Tabla 2: Distribución de la población según tipo de seguro, turno de servicio, días de hospitalización, área de servicio y complejidad del paciente $(n=384)$

\begin{tabular}{lcc}
\hline Variable & $\begin{array}{c}\text { Frecuencia } \\
\text { N }\end{array}$ & Porcentaje \% \\
\hline Tipo de Seguro & & \\
\hline Campesino & 25 & $6,5 \%$ \\
General & 326 & $84,9 \%$ \\
Montepío & 8 & $2,1 \%$ \\
Jubilado & 25 & $6,5 \%$ \\
\hline Turno de servicio & & \\
\hline Matutino & 118 & $30,7 \%$ \\
Vespertino & 151 & $39,4 \%$ \\
Nocturno & 115 & $29,9 \%$ \\
\hline Días de Hospitalización & & \\
\hline Dos & 11 & $2,9 \%$ \\
Tres & 150 & $39,1 \%$ \\
Cuatro & 90 & $23,4 \%$ \\
Más de Cuatro & 133 & $34,6 \%$ \\
\hline Área de Servicio & & \\
\hline Clínica 1 & 50 & $13,0 \%$ \\
Clínica 2 & 41 & $10,7 \%$ \\
Clínica 3 & 42 & $10,9 \%$ \\
Cardiología & 32 & $8,3 \%$ \\
Cirugía General & 60 & $15,7 \%$ \\
Neurología & 39 & $10,2 \%$ \\
Traumatología & 35 & $9,1 \%$ \\
Cirugía Oncológica & 38 & $12,2 \%$ \\
Ginecología & 47 & \\
\hline
\end{tabular}

Fuente: Base de datos SPSS

Elaborado por: Autores
En la tabla 3 se evidencia que en relación a la complejidad baja del paciente tenemos el 61,5\%, mientras que en la complejidad intermedia de los pacientes hospitalizados es de $38,5 \%$.

Tabla 3: Distribución de la población según la complejidad del paciente $(n=384)$

\begin{tabular}{lcc}
\hline Variable & $\begin{array}{c}\text { Frecuencia } \\
\text { N }\end{array}$ & Porcentaje \% \\
\hline Complejidad del Paciente & & \\
\hline Complejidad Baja & 236 & $231(61,5 \%)$ \\
Complejidad Intermedio & 148 & $143(38,5 \%)$ \\
\hline
\end{tabular}

\section{Fuente: Base de datos SPSS}

Elaborado por: Autores

En la tabla 4 se puede observar que, las seis categorías del trato humanizado prevalecen un nivel de siempre percibe trato humanizado y tan solo en las categorías dar apoyo emocional y empatía se percibió valores mínimos en el nivel a veces percibe trato humanizado.

Tabla 4: Percepción de trato humanizado según sus categorías $(\mathrm{n}=384)$

\begin{tabular}{lcccccc}
\hline \multirow{2}{*}{ Variable } & \multicolumn{2}{c}{ Siempre } & \multicolumn{2}{c}{ Casi siempre } & \multicolumn{2}{c}{ A veces } \\
\cline { 2 - 7 } & $\mathrm{N}$ & $\%$ & $\mathrm{~N}$ & $\%$ & $\mathrm{~N}$ & $\%$ \\
\hline Dar apoyo emocional & 354 & $92,2 \%$ & 28 & $7,3 \%$ & 2 & $0,5 \%$ \\
Dar apoyo físico & 370 & $96,4 \%$ & 14 & $3,6 \%$ & 00 & 00 \\
Empatía & 206 & $53,6 \%$ & 173 & $45,1 \%$ & 5 & $1,3 \%$ \\
Cualidades del hacer & 364 & $94,8 \%$ & 20 & $5,2 \%$ & 00 & 00 \\
Proactividad & 248 & $64,6 \%$ & 136 & $35,4 \%$ & 00 & 00 \\
\hline
\end{tabular}

Fuente: Base de datos SPSS

Elaborado por: Autores

En la tabla 5 se obtuvo que mayor parte de los encuestados dieron una respuesta de siempre reciben un trato humanizado con un porcentaje de 97,4\%, mientras que el $2,6 \%$ dieron una respuesta de casi siempre perciben un trato humanizado.

Tabla 5: Representación del puntaje global del trato humanizado $(n=384)$

\begin{tabular}{lcc}
\hline Variable & $\begin{array}{c}\text { Frecuencia } \\
\text { N }\end{array}$ & Porcentaje \% \\
\hline Complejidad del Paciente & & \\
\hline Siempre & 374 & $97,4 \%$ \\
Casi Siempre & 10 & $2,6 \%$ \\
Total & 384 & $100 \%$ \\
\hline
\end{tabular}

Fuente: Base de datos SPSS

Elaborado por: Autores
Al tener tendencia a una alta percepción de trato humanizado y necesitar 2 grupos para el análisis de asociación se agrupo la variable trato humanizado en dos categorías que son: trato humanizado óptimo, en él se ubican las personas con una percepción siempre, y el segundo grupo se lo denomina trato humanizado regular, en el cual se ubican las personas con una percepción de casi siempre.

En la tabla 6 se observan los factores de riesgo analizados en el presente estudio, donde se consideró el tipo de seguro como factor de riesgo, se encontró que este grupo de análisis el riesgo es de 6,85 (IC 95\%: 1.658 - 28,353 valor p: 0,002). 
Tabla 6: Percepción de trato humanizado según sus categorías $(n=384)$

\begin{tabular}{|c|c|c|c|c|c|}
\hline Variable & $\begin{array}{c}\text { Trato humanizado } \\
\text { regular }\end{array}$ & $\begin{array}{c}\text { Trato humanizado } \\
\text { optimo }\end{array}$ & OR & IC & $\mathbf{p}$ \\
\hline \multicolumn{6}{|l|}{ Edad } \\
\hline Adulto & $10(2,6 \%)$ & $352(91,7 \%)$ & \multirow{2}{*}{0,972} & \multirow{2}{*}{$0,956-0,989$} & \multirow{2}{*}{0,430} \\
\hline Adulto mayor & $0(0,0 \%)$ & $22(5,7 \%)$ & & & \\
\hline \multicolumn{6}{|l|}{ Sexo } \\
\hline Hombres & $6(1,6 \%)$ & $208(54,2 \%)$ & \multirow{2}{*}{1,197} & \multirow{2}{*}{$0,332-4,312$} & \multirow{2}{*}{0,783} \\
\hline Mujeres & $4(1,0 \%)$ & $166(43,2 \%)$ & & & \\
\hline \multicolumn{6}{|l|}{ Estado civil } \\
\hline Con pareja & $7(1,8 \%)$ & $277(72,1 \%)$ & \multirow{2}{*}{0,817} & \multirow{2}{*}{$0,207-3,222$} & \multirow{2}{*}{0,773} \\
\hline Sin Pareja & $3(0,8 \%)$ & $97(25,3 \%)$ & & & \\
\hline \multicolumn{6}{|l|}{ Nivel de instrucción } \\
\hline No Profesionales & $10(2,6 \%)$ & $336(87,5 \%)$ & \multirow{2}{*}{0,971} & \multirow{2}{*}{$0,954-0,989$} & \multirow{2}{*}{0,288} \\
\hline Profesionales & $0(0,0 \%)$ & $38(9,9 \%)$ & & & \\
\hline \multicolumn{6}{|l|}{ Etnia } \\
\hline Mestizo & $10(2,6 \%)$ & $370(96,4 \%)$ & \multirow{2}{*}{0,974} & \multirow{2}{*}{$0,958-0,90$} & \multirow{2}{*}{0,742} \\
\hline Otros & $0(0,0 \%)$ & $4(1,0 \%)$ & & & \\
\hline \multicolumn{6}{|l|}{ Tipo de Seguro } \\
\hline Seguro IESS & $3(0,8 \%)$ & $22(5,7 \%)$ & \multirow{2}{*}{6,857} & \multirow{2}{*}{$1.658-28,353$} & \multirow{2}{*}{0,002} \\
\hline Otros seguro & $7(1,8 \%)$ & $352(91,7 \%)$ & & & \\
\hline \multicolumn{6}{|l|}{ Turno de servicio } \\
\hline Mañana & $3(0,8 \%)$ & $115(29,9 \%)$ & \multirow{2}{*}{0,965} & \multirow{2}{*}{$0,245-3,799$} & \multirow{2}{*}{0,960} \\
\hline Otros & $7(1,8 \%)$ & $259(67,5 \%)$ & & & \\
\hline Vespertino & $3(0,8 \%)$ & $148(38,5 \%)$ & \multirow{2}{*}{0,654} & \multirow{2}{*}{$0,167-2,571$} & 0541 \\
\hline Otros & $7(1,8 \%)$ & $226(58,9 \%)$ & & & 0,541 \\
\hline Nocturno & $4(1,0 \%)$ & $111(28,9 \%)$ & & & \\
\hline Otros & $6(1,6 \%)$ & $263(68,5 \%)$ & 1,580 & $0,437-5,707$ & 0,482 \\
\hline Días de Hospitalización & & & & & \\
\hline 72 horas & $3(1,9 \%)$ & $158(98,1 \%)$ & & & \\
\hline Mayor a 72 horas & $7(3,1 \%)$ & $216(96,9 \%)$ & 0,586 & 0,149 & 0,439 \\
\hline Complejidad del pacien & & & & & \\
\hline Complejidad baja & $5(1,3 \%)$ & $231(60,2 \%)$ & & & \\
\hline Complejidad intermedia & $5(1,3 \%)$ & $143(37,2 \%)$ & 0,619 & $0,18-2,17$ & 0,451 \\
\hline
\end{tabular}

Fuente: Base de datos SPSS

Elaborado por: Autores

\section{Discusión}

El arte de cuidar ha ido evolucionando considerando a la persona como ser humano y utilizando las responsabilidades éticas, sociales y profesionales es por ello que la práctica de enfermería debe estar basada en un sistema de valor humano conjuntamente con la parte científica y de esta manera ayudar a la recuperación de la salud de los pacientes. Siguiendo los objetivos planteados en la investigación se discuten los siguientes resultados. ${ }^{11}$

En relación a la variable edad indico que la edad de los pacientes hospitalizados se ubica entre 35 a 65 años (Adulto) con el $62,8 \%$, la edad mínima que se encontró fue de 20 y la edad máxima fue 85 años, estos datos se contraponen al estudio realizado de Echevarría SH, et al. Percepción del paciente sobre calidad del cuidado humanizado brindado por la enfermera en una clínica de Lima en 2017, en donde la edad que prevaleció fue de 32,4\% (de 20 a 29 años) siendo adultos jóvenes. En base a los resultado se pudo establecer que no tiene una relación directa entre los dos estudios. ${ }^{11}$
En base a la variable sexo el predominio de ingreso en esta institución es de 55,7 \%, mientras que el sexo femenino corresponde a un 44,3\%, presentando así una relación con el estudio de Pat ME, et al., realizado en México en el año 2017 sobre la Satisfacción del paciente adulto hospitalizado, dando los siguientes datos el $57 \%$ correspondía al género masculino y el $43 \%$ al femenino, en los dos estudios existe una similitud de ingresos de los pacientes de sexo masculino. $^{12}$

En el estado civil los pacientes con pareja (unión libre y casados) tienen un porcentaje de $73,9 \%$, mientras que pacientes sin pareja (solteros, divorciados) el 26,1\%, presentando así una diferencia significativa con relación al estudio de Massa RE, et al. En Colombia en el 2016, el estudio que buscó establecer la relación entre cuidado humanizado por enfermería con la hospitalización de pacientes, prevaleció la "unión libre" con $31.8 \%$, seguida de "soltero" con el $29.5 \%$, y en último lugar los "divorciados", con solo el $2.3 \% .^{13}$ 
En cuanto a la etnia predominan los mestizos con el mayor porcentaje del 98,9\% (380 usuarios), afro ecuatoriano e indígena con $0,3 \%$, se pueden observar datos contrastantes en comparación con el estudio realizado de Monje P, et al., en el 2018 sobre la percepción de cuidado humanizado de enfermería desde la perspectiva de usuarios hospitalizados, en Chile en donde el $88,3 \%$ eran mestizos y el $11,7 \%$ se consideran parte de la etnia mapuche u otras etnias; esto podría deberse a que el IESS brinda atención prioritaria a personas que tienen dependencia laboral, y la población indígena principalmente se ubica en las regiones rurales del país y se dedican a actividades agrarias. ${ }^{11}$

En la variable turno de servicio, en la jornada laboral del turno vespertino los usuarios que son atendidos y encuestados son el 39,4\%, y en la jornada laboral nocturna se obtuvo que los pacientes que se atienden son el $29,9 \%$, existiendo una semejanza en el turno vespertino con la presente investigación, con relación al estudio de, Santos M, et al., en el 2015 sobre percepción y cumplimiento del trato digno como indicador de calidad en la atención de enfermería en derecho habientes de una institución de salud, en México, revela en su estudio que en el turno vespertino los pacientes atendidos son el $30 \%$, y del turno nocturno son el $17 \%$, cabe mencionar que en el turno nocturno existe una diferencia debido a diferentes factores ya sea por la capacidad del hospital en el cual se realizó la presente investigación, ubicación del recinto hospitalario entre otra las cuales hacen que este valor difiera. ${ }^{14}$

En lo que respecta a la percepción de Trato Humanizado el instrumento PCHE consta de 32 ítems donde la mayoría de los usuarios encuestados otorgó el máximo puntaje estableciendo que siempre perciben trato humanizado $(97,4 \%)$, y el 2,6\% casi siempre. En comparación con el estudio de Monje P, et al., en el 2018 sobre la percepción de cuidado humanizado de enfermería desde la perspectiva de usuarios hospitalizados, en Chile se observa que existe semejanza con el presente estudio ya que, las categorías de siempre ( $86 \%$ ) y casi siempre $(11,7 \%)$, el $(1.8 \%)$ algunas veces y el $(0,6 \%)$ nunca, Es decir demuestran que los pacientes perciben que los profesionales de enfermería se toman el tiempo para aplicar técnicas o procedimientos, además demuestran que el cuidado humanizado es una labor fundamental de enfermería que se ve reflejada en su labor diaria. ${ }^{11}$

En virtud de los resultados obtenidos, se puede indicar que la percepción que tienen los pacientes en relación al trato humanizado y los factores socio demográficos, no corresponde a una asociación significativa, considerando que los resultado obtenidos fueron inferiores ( $p>0.05$ ); de manera similar lo demuestra un estudio realizado en Lima por Echevarría A, et al., en el año 2018 sobre: Relación Entre Percepción Del Cuidado Humanizado de Enfermería y Factores Sociodemográficos, el cual dio como resultado que la asociación fue inferior a $(\mathrm{p}>0.05) .{ }^{15}$

En relación a la variable área de servicio y días de hospitalización asociada con el trato humanizado no existe una asociación significativa, de manera similar con el es- tudio de Massa RE, et al., En Colombia en el 2016, sobre Relación entre Cuidado Humanizado por Enfermería con la Hospitalización de Pacientes, La percepción de cuidado humanizado relacionado con la especialidad médica del servicio, tiempo actual de estancia hospitalaria y hospitalizaciones previas, con una $\mathrm{p}>0.05$; por consiguiente no existe una significancia con estas dos variables. ${ }^{13}$

En cuanto a la relación de la variable trato humanizado asociada con el tipo de seguro se encontró asociación, obteniendo los siguientes datos (OR: 6,857 IC: 1.658 28,353 p: 0,002), en relación al estudia de Rosas PE, et al., sobre el Trato Adecuado respuesta del sistema mexicano de salud a las expectativas no medicas de sus usuarios. En México entre los individuos encuestados, $56 \%$ reportaron no tener ningún tipo de seguro médico; $32 \%$ del total informaron estar asegurados en el Instituto mexicano del seguro social (IMSS), y el $41 \%$ de los informantes manifestaron recibir servicios medico a través de uno de los sub sistemas de seguridad social (incluido en IMSS). Solo el 1,5\% de los entrevistados manifestó tener algún tipo de seguro voluntario. La suma es mayor de $100 \%$ debido a que el $1,5 \%$ de los entrevistados refirieron tener más de un seguro. ${ }^{16}$

\section{Conclusión}

Con la aplicación del instrumento de PCHE se pudo obtener información valiosa sobre la percepción del Trato Humanizado por parte de los profesionales de enfermería; los puntajes globales determinaron que el 97, $4 \%$ de los usuarios estuvieron satisfechos con el trato brindado, al momento del análisis de las categorías los resultados con mayor puntuación fueron siempre, lo que indica que el personal es capacitado y apto para brindar una buena atención.

En base a los resultados obtenidos sobre las variables edad, sexo, estado civil se concluye que en el Hospital José Carrasco Arteaga la mayor parte de usuarios hospitalizados fueron adultos en un 62,8\% comprendido entre las edades de 35 a 65 años, por otra parte, con un 55,7\% la atención fue mayor a personas de sexo masculino, el $57,3 \%$ eran casados y en relación a la etnia fue $98,9 \%$ mestizos.

Como conclusión final tenemos que no existe asociación entre la percepción del trato humanizado y las siguientes variables edad, sexo, estado civil, nivel de instrucción, etnia, residencia, turno de servicio, días de hospitalización, áreas de servicio, complejidad del paciente con el único factor que se asoció fue el tipo de seguro, siendo así que tener un seguro campesino disminuye la percepción de trato humanizado.

\section{Fuente de Financiamiento}

Este estudio es autofinanciado.

\section{Conflicto de Intereses}

No existen conflictos personales, profesionales, financieroso de otro tipo. 


\section{Consentimiento Informado}

Los autores cuentan con el consentimiento informado de los pacientes para la investigación, la publicación del caso y sus imágenes.

\section{Referencias Bibliográficas}

1. Elers Mastrapa Y, Gibert Lamadrid M del P. Relación enfermera-paciente: una perspectiva desde las teorías de las relaciones interpersonales. Rev Cuba Enfermería. Diciembre de 2016; 32(4):0-0. Disponible en: http://scielo.sld.cu/scielo.php?script=sci_arttext\& pid=S0864-03192016000400019

2. De Arco-Canoles ODC, Suarez-Calle ZK. Rol de los profesionales de Enfermería en el sistema de salud colombiano. Univ Salud. 30 de abril de 2018; 20(2):171. Disponible en: http://www.scielo.org.co/pdf/reus/v20n2/ 0124-7107-reus-20-02-00171.pdf

3. Gómez L, Isabel M. El cuidado de enfermería a los grupos humanos. Rev Univ Ind Santander Salud. Agosto de 2015; 47(2):209-13. Disponible en: http://www.scielo.org.co/scielo.php?script=sci_arttext\& pid=S0121-08072015000200013

4. Ramos Guajardo S, Ceballos Vásquez P. Cuidado humanizado y riesgos psicosociales: una relación percibida por profesionales de enfermería en Chile. Enferm Cuid Humaniz [Internet]. 23 de marzo de 2018 [citado 6 de febrero de 2020];7(1). Disponible en: http://www.scielo. edu.uy/pdf/ech/v7n1/2393-6606-ech-7-01-3.pdf

5. Hernández Terrazas LE, Díaz Oviedo A, Martínez Licona JF, Gaytan Hernandez D, Hernández Terrazas LE, Díaz Oviedo A, et al. Nursing Education in Humanized care. Esc Anna Nery [Internet]. 2018 [citado 7 de Febrero de 2020]; 22(1). Disponible en: http://www.scielo.br/scielo.php?script=sci_arttext\&pid= S1414-81452018000100219\&lng=en\&tlng=en

6. Guerrero-Ramírez R, Meneses-La Riva ME, De La Cruz-Ruiz M. Cuidado Humanizado de enfermería según la teoría de Jean Watson, servicio de medicina del Hospital Daniel Alcides Carrión. Lima- Ca1lao, 2015. Rev Enfermeria Hered. 20 de febrero de 2017;9(2):125. Disponible en: https://faenf.cayetano.edu. pe/images/2017/revistavo19/9.pdf

7. Landman Navarro C, Cruz Osorio MJ, García García E, Pérez Meza P, Sandoval Barrera P, Serey Burgos K, et al. Satisfacción Usuaria Respecto a Competencia de Comunicación del 'Profesional de Enfermería. Cienc Enferm. Abril de 2015;21(1):91-102. Disponible en: https://scielo. conicyt.cl/pdf/cienf/v21n1/art_09.pdf

8. Manual_MAIS-MSP12.12.12.pdf [Internet]. [Citado 6 de febrero de 2020]. Disponible en: http://instituciones.msp.gob.ec/somossalud/images/ documentos/guia/Manual_MAIS-MSP12.12.12.pdf

9. Monje V. P, Miranda C. P, Oyarzün G. J, Seguel P. F, Flores G. E, Monje V. P, et al. Percepción De Cuidado Humanizado De Enfermería Desde La Perspectiva De Usuarios Hospitalizados. Cienc Enferm [Internet]. 2018
[Citado 7 de Febrero de 2020];24. Disponible en: https:// scielo.conicyt.cl/pdf/cienf/v24/0717-9553-cienf-24-5.pdf 10. Vargas BZ, Porras MX, Legister TG, Benavides CM. Valoración de la escala (Perroca) para la categorización de pacientes. Enferm En Costa Rica. Marzo de 2016;34(2). Disponible en: http://enfermeria.cr/ revista/docs/Valoracion_de_la_Escala_Perroca_para_ la_categorizacion_de_pacientes_en_el_Hospital_Rafael_ Angel_Calderon_Guardia_Octubre_del_2013.pdf

1. Schmidt HE. Percepción del paciente sobre calidad del cuidado humanizado brindado por la enfermera en una clínica-Lima. Cuid Salud Kawsayninchis.2017;3(1):23948. Disponible en: http://revistas.urp.edu.pe/index.php/ Cuidado_y_salud/article/view/1421/1316

2. Social R de E del IM del S. Open Journal Systems. [Citado 7 de Febrero de 2020]; Disponible en: http://revistaenfermeria.imss.gob.mx/editorial/index. php/revista_enfermeria/article/view/110

3. Romero Massa E, Contreras Méndez IM, Universidad de Cartagena, Moncada Serrano A, Universidad de Cartagena. Relación Entre Cuidado Humanizado Por Enfermería Con La Hospitalización De Pacientes. Hacia Promoc Salud. 5 de junio de 2016;0121-7577(24628425):26-36.Disponible en: http://www.scielo.org.co/pdf/ hpsal/v21n1/v21n1a03.pdf

4. Percepción y cumplimiento del trato digno como indicador de_calidad en la atención de enfermería en derechohabientres de_una institución de salud. [Citado 7 de Febrero de 2020].Disponible en: https://www.redalyc.org/ pdf/4578/457844966004.pdf?fbclid=IwAR3ySw93nr5L2wNrJ54ylei7xJNWL4mxcyUO09iOejtPGjIt5DPtiTUiRg

15. Relación Entre Percepción del Cuidado Humanizado de Enfermería y Factores Sociodemográficos, en Pacientes Hospitalizados en un Hospital Público de LimaEchevarría Anyosa, Luz María.pdf [Internet]. [Citado 4 de marzo de 2020]. Disponible en: http://repositorio.uwiener. edu.pe/bitstream/handle/123456789/1477/TITULO\%20$\% 20$ Echevarr\%c3\%ada\%20Anyosa\%2c\%20Luz\% $20 \mathrm{Mar} \% \mathrm{c} 3 \%$ ada.pdf? sequence $=1 \&$ is Allowed $=\mathrm{y}$

6. Puentes-Rosas E. Trato adecuado: respuesta del sistema mexicano de salud a las expectativas no médicas de sus usuarios. Salud Pública México [Internet]. 2 de febrero de 2005 [citado 4 de marzo de 2020];47(0). Disponible en: http://saludpublica.mx/index.php/spm/article/view/4684

Recibido: 9 de octubre de 2020

Aceptado: 25 de octubre de 2020 
